\title{
Ocronosis: presentación de un caso con afectación multiorgánica incluido pericardio
}

\author{
F. J. COBOS SOLER, R. MOLERO CABRILLA \\ Servicio de Medicina Interna. Hospital Los Morales. Córdoba
}

\author{
OCHRONOSIS: REPORT OF A CASE WITH MULTISYSTEMIC \\ AFFECTATION, INCLUDING PERICARDIUM
}

\begin{abstract}
RESUMEN
La Alcaptonuria es una rara enfermedad hereditaria del catabolismo de la tirosina, autosómico recesiva, caracterizada por la deficiencia en la enzima homogentísico 1,2 oxigenasa. Este déficit enzimático produce la eliminación continuada de ácido homogentísico en orina (Alcaptonuria) y la acumulación de pigmento de ácido homogentísico en el tejido conectivo (Ocronosis). Los rasgos clínicos más característicos son la emisión de una orina que se torna de color negro-pardusca al contacto con el aire; pigmentación oscura en escleras y piel; calcificación de los discos intervertebrales y afectación cardiovascular sobretodo estenosis de la válvula aórtica. El diagnóstico se confirma mediante la detección de ácido homogentísico en orina.

Presentamos el caso de una mujer de 87 años de edad con los mismos datos clínicos reflejados anteriormente y con calcificación pericárdica, no habiéndose recogido hasta el momento en la literatura similar alteración en estos pacientes.
\end{abstract}

PALABRAS CLAVE: Ocronosis. Alcaptonuria. Artropatía. Pericardio.

\begin{abstract}
Alkaptonuric ochronosis is rare disorder of tyrosin catabolism with an autosomal recessive trait. Alkaptonuric patients are deficient for homogentisate 1,2- dioxygenase. This enzymatic deficiency leads to the elimination of large amounts of homogentisic acid in the urine (Alkapto nuria) with accumulation of homogentisic acid oxidized pigment in the connective tissue (Ochronosis). The most common clinical features are dark brown discoloration of urine on exposure to air; ocular and cutane ous pigmentation; calcification of the intervertebral disc and cardiovas cular ochronosis, especially calcification and stenosis of the aortic valve. The diagnosis is confirmed by detection of homogentisic acid in urine.

We report a case of a 87 year old female which has all these clinical features mentioned above and pericardiac calcification, which had not been previously reported, to our knowledge.
\end{abstract}

KEY WORDS: Alkaptonuria. Ochronosis. Arthropathy. Pericardium.

Cobos Soler FJ, Molero Cabrilla R. Ocronosis: presentación de un caso con afectación multiorgánica incluido pericardio. An Med Interna (Madrid) 2002; 19: 583-585.

\section{INTRODUCCIÓN}

La ocronosis puede ser definida como el conjunto de manifestaciones musculoesqueléticas de la Alcaptonuria. Los pacientes alcaptonúricos que viven hasta la cuarta década de la vida, casi invariablemente presentan ocronosis.

Presentamos el caso de una mujer de ochenta y siete años de edad con intensas manifestaciones ocronóticas facilitadas por el depósito continuo, durante muchos años, de material ocronótico en sus tejidos.

Se trata, por tanto, de uno de los casos de mayor edad descrito en la literatura, lo que explicaría, que localizaciones no registradas hasta el momento, como el pericardio, se vean afectadas en esta paciente.

\section{CASO APORTADO}

Paciente de 87 años de edad, remitida a nuestro Centro para rehabilitación, tras haberle implantado una prótesis parcial de cadera por fractura subcapital derecha.

Desde su ingreso y, debido al sondaje vesical al que fue sometida, se apreciaron orinas oscuras. Valorada por el servicio de Urología, se descartó patología orgánica relevante de dicha especialidad.

Clínicamente la paciente se encontraba asintomática. Destacaba en la anamnesis, la presencia de episodios repetidos de dolor lumbar de características mecánicas que no habían sido estudiados hasta el momento, así como una marcada hipoacusia.

En la exploración general se evidenció una coloración oscura en escleróticas (Fig. 1), pabellones auriculares, membranas timpánicas y dorso de las manos. Además la paciente presentaba una rigidez y

Trabajo aceptado: 20 de septiembre de 2001

Correspondencia: Fco. J. Cobos Soler. C/ Escritor López de Cárdenas 5, 3º-1. 14007 Córdoba 


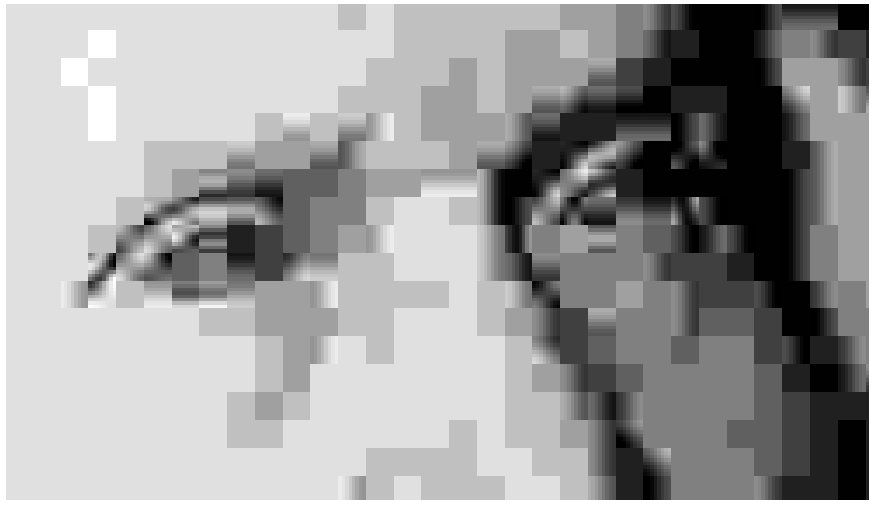

Fig. 1. Coloración oscura de escleróticas.

encorvamiento de tronco hacia delante, que le confería un porte típicamente anquilótico.

Los valores de la hematimetría, bioquímica y sedimento de orina resultaron normales.

En las radiografías simples de tórax y abdomen (Fig. 2), se observaron calcificaciones de partes blandas a nivel de mamas, calcificación de discos intervertebrales, troncos supraaórticos y de pericardio.

Se determinó el ácido homogentísico en orina de 24 h por cromatografía líquida de alta resolución que mostró un valor de 2.683 $\mathrm{mg} / 24 \mathrm{~h}$, siendo el valor de referencia menor de $10 \mathrm{mg} / 24 \mathrm{~h}$.

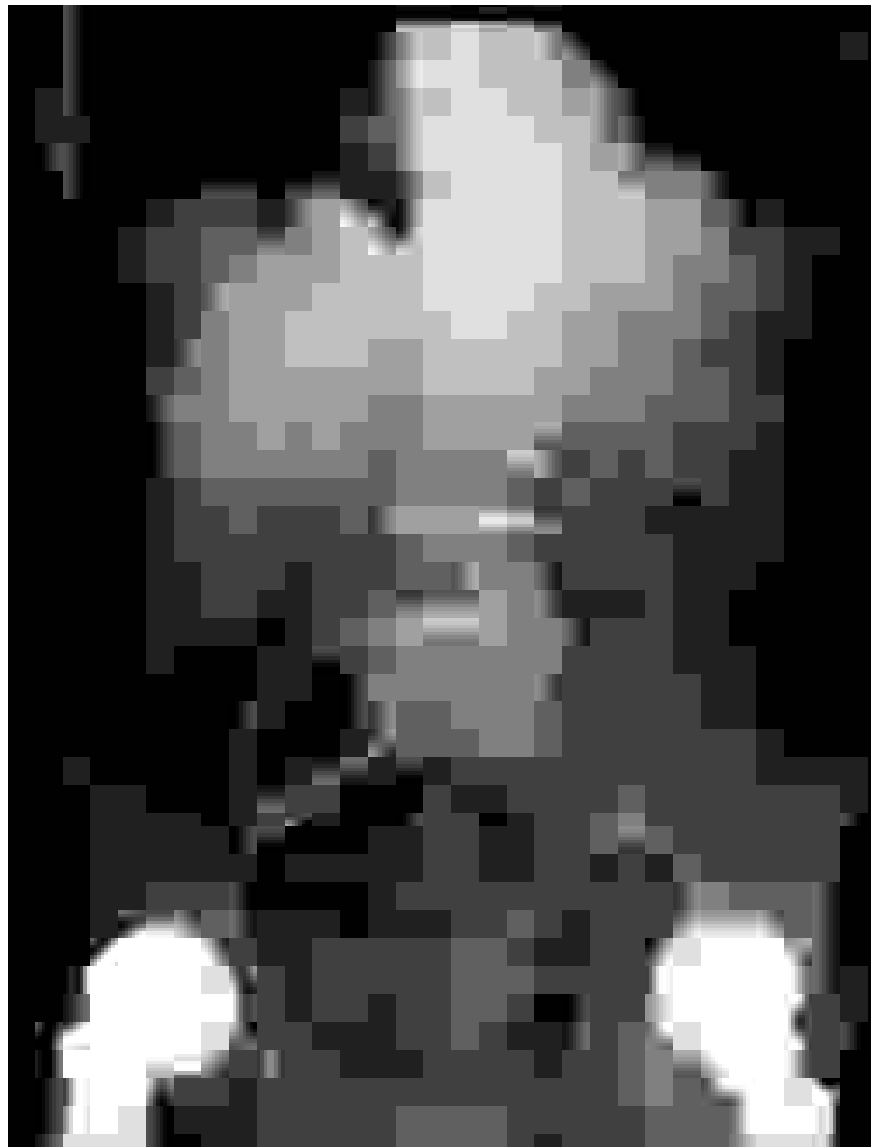

Fig. 2. Calcificaciones de partes blandas, discos intervertebrales.

\section{DISCUSIÓN}

La Alcaptonuria es una rara enfermedad hereditaria del catabolismo de la tirosina, autosómico recesiva, caracterizada por la presencia de ácido homogentísico en orina. Su prevalencia aproximada es de 1/1.000.000 de habitantes (2). Fue la primera alteración en ser interpretada como un error congénito del metabolismo.

Estos pacientes, tienen deficiencia en la homogentísico 1,2 oxigenasa (HGO), enzima encargada de transformar el ácido homogentísico en ácido maleilacetoacético, originando depósito del primero en diversos tejidos del organismo ricos en colágeno (Ocronosis). Se han identificado, hasta 16 tipos de mutaciones en el gen HGO humano (cromosoma 3), que pueden dar lugar a una pérdida de función de la enzima (3). También se ha relacionado con la presencia de HLA B-27 positivo.

La alcaptonuria da lugar a la aparición de una orina de color negro-pardusca, como consecuencia de la oxidación del ácido homogentísico que contiene. Los pacientes alcaptonúricos que viven hasta la cuarta década de la vida, casi invariablemente presentan ocronosis.

Consideramos, que el mayor interés clínico de la ocronosis, reside en la peculariedad de sus características clínicas y radiológicas, siendo alguna de ellas patognomónicas de la enfermedad.

Clínicamente, están descritos un amplio espectro de manifestaciones ocronóticas destacando la presencia de una pigmentación oscuro-azulada en pabellones auriculares, regiones malares y escleróticas (Fig. 1). A menudo el cerumen del conducto auditivo externo es negro y la periferia de la membrana timpánica es gris oscura, desembocando, a veces, en un deterioro de la audición, sobretodo en casos de edad avanzada como el descrito en el presente artículo.

Los pacientes ocronóticos, con el paso del tiempo, van a desarrollar un cuadro de espondilosis ocronótica, caracterizado por el envaramiento progresivo de la columna lumbar y pérdida de la lordosis fisiológica, casi siempre de forma sintomática. Especialmente significativo resulta el historial de lumbalgias no filiadas que presentaba nuestra paciente.

Radiográficamente, resulta patognomónica la presencia de calcificación y osificación de los discos intervertebrales (Fig. 2), pudiendo aparecer también unas articulaciones sacroiliacas no fusionadas y una osteofitosis desproporcionalmente escasa. En algunas ocasiones, padecen artropatías periféricas, siendo las rodillas, los hombros y las caderas las articulaciones más comúnmente afectadas. El líquido sinovial contiene pequeñas cantidades de ácido homogentísico y no tiene características inflamatorias.

Se ha descrito, algún caso con afectación de la inmunidad celular y la consiguiente aparición de infecciones bacterianas recurrentes. La respuesta humoral y la función de los neutrófilos, no están alteradas (4).

En una gran proporción de hombres con ocronosis aparecen cálculos prostáticos de color negro (5).

Finalmente, el examen ecocardiográfico de estos enfermos, ha revelado la aparición de calcificaciones valvulares, sobretodo en válvula aórtica, que han sido relacionados con el depósito de material ocronótico (2). Soplos cardiacos fuertes, sobretodo sistólicos, se han descrito en casi un 15-20\% de los pacientes con ocronosis. No hemos encontrado en la revisión de la literatura realizada ninguna referencia sobre afectación pericárdica en esta enfermedad. 
El diagnóstico de ocronosis se confirma mediante la detección de ácido homogentísico en orina de 24 horas. En nuestro caso, el análisis mostró valores exageradamente elevados.

\section{Bibliografía}

1. Schumacher, H. R. Ocronosis, Hemocromatosis y enfermedad de Wilson. In: McCarty, D. J. editor. Artritis y otras patologías relacionadas. $10^{\mathrm{a}}$ ed. Buenos Aires: Editorial médica panamericana; 1987, p. 1577-82.

2. Kocyigit H, Gurgan A, Terzioglu R, Gurgan U. Clinical, Radiographic and Echocardiographic Finding In a patient with ochronosis. Clin Rheumatol. 1998; 17: 403-6.

3. Beltran-Valero de Bernabe D, Granadino B, Chiarelli I, Porfirio B Mayatepek E, Aquaron R et al. Mutation And Polymorphism Análisis Of The human homogentisate 1,2-dioxygenase gene in alkaptonuria patients. Am J Hum Genet. 1998; 62: 776-84.

4. Mori S, Kawaguchi T, Kakinuma H, Fuji-I H, Koga S, Suzushisma H et al. Alcaptonuria: A case Complicated with Valvular heart disease and immunodeficiency. Intern Med. 1994; 33: 512-6.

5. Concepción Masip T, Banares Baudet F, Traba ML, Rodríguez de Minon Cifuentes JL. Alkaptonuria, Prostatic calculi, And Ectopic ureter. Actas Urol Esp. 1997; 21: 167-70.
Hemos informado, en esta ocasión, sobre un caso de ocronosis donde se evidencia una clara afectación pericárdica, no habiéndose recogido hasta el momento en la literatura similar alteración.

6. Simianer S, Krause D, Rau R.Concomitant Manifestation Of Ochronosis And Chronic polyarthritis in a patient. Z Rheumatol. 1998; 57: 50-2.

7. Gutzmer R, Herbst RA, Kiehl P, Kapp A, Weiss J. Alkaptonuric Ochronosis Report Of Two Affected brothers. J Am Acad Dermatol. 1997; 37: 305-7.

8. Corra T, Zaccala M, Galante M. Ochronosic Arthropathy: Rapid Destructive Hip Osteoarthritis associated with metabolic disease. Clin Rheumatol. 1995; 14: 474-7.

9. Kabasakal Y; Kiyici I, Ozmen D, Yagci A, Gusmidis G. Spinal Abnormalities Similar To An kilosing Spondylitis in a 58 year old woman with ochronosis. Clin Rheumatol. 1995; 14: 355-7.

10. Cortina R, Moris C, Astudillo A, Gosalbez F, Cortina A. Familial Ochronosis. Eur Heart J. 1995; 16: 285-6.

11. Quaterman M, Hall JM Jr., Gouridin Fw, ChalkerDk. Photodistributed Hereditary Ochronosis. Arch Dermatol. 1992; 128: 1657-8

12. Albers SE, Brozena SJ, Glass LF, Fenske NA. Alkaptonuria And Ochronosis: Case report And review. J Am Acad Dermatol. 1992; 27: 609-14. 